\title{
TURKOPHILIE AM WIENER HOF MARIA THERESIAS
}

\author{
BRIGITTA PESTI \\ Universität Wien, Abteilung für Finno-Ugristik \\ brigitta.pesti@univie.ac.at
}

\begin{abstract}
In dem Beitrag geht es um die veränderte Rezeption der Osmanen im Zuge der Rückeroberung weiter europäischer Gebiete aus ihrer Oberhoheit. Türkische Mode, türkische Sitten und Musik wurden europaweit modern und waren sehr gefragt. Auch der Wiener Hof übernahm diesen Modestil und selbst Maria Theresa ließ sich wiederholt in türkischer Kleidung von ihren Lieblingsmalern porträtieren. Selbst im Repräsentationsbereich liebte man das Dekor alla turca. Im östlicheren Teil der Habsburger Monarchie blieb das Bild des ehemaligen Erzfeindes allerdings weiterhin ambivalent.
\end{abstract}

Schlüsselwörter: Turkophilie, Wiener Hof, Repräsentation, Festlichkeiten, Bildende Künste, Maria Theresia

\section{Neue Ansätze in der Türken-Rezeption}

Nach beinahe drei Jahrhunderten, in denen die Beziehungen zwischen der Habsburgermonarchie und dem Osmanischen Reich durch Kriege bestimmt gewesen waren, setzte nach der osmanischen Niederlage bei der zweiten Belagerung Wiens (1683) und dem schließlich erzielten Frieden von Karlowitz (1699) eine Entspannung ein, die hauptsächlich in Folge eines kulturellen und wissenschaftlichen Austausches, sowie intensiver politischer und wirtschaftlicher Beziehungen ein zunehmendes Interesse an den Fremden ausgelöst hatte.

Das seit dem Mittelalter überlieferte polarisierende und dämonisierende Bild vom Orient wurde nun von vielen Intellektuellen der Zeit neu beleuchtet, und so wandelte sich das Bild der Osmanen vom bedrohlichen „Bluthund“, vom Schrekken der Christenheit zum exotischen Fremden. ${ }^{1}$ Dieser kulturelle Wandel ging von Ländern im westlichen Europa, wie England, den Niederlanden oder vor allem von Frankreich aus, die von der Türkengefahr ideell oder materiell weniger oder kaum betroffen waren. Die türkenfreundliche Politik Frankreichs unter Ludwig XIV. und die hauptsächlich auf wirtschaftliche Interessen ausgerichteten Beziehungen der Niederlande und Englands zum Osmanischen Reich, sowie das Fehlen einer türkischen Bedrohung im öffentlichen Bewusstsein führten in diesen Staaten zu gesellschaftlichen, politischen und kulturellen Veränderungen, 
woraus bereits im 16.-17. Jahrhundert eine bessere Beziehung zum Orient entstand. ${ }^{2}$

Die neue, vorurteilsfreie Beziehung spiegelte sich zuerst in westeuropäischen Reisebeschreibungen wider, in denen anstatt des früheren Feindbilds eine unvoreingenommene und analytische Berichterstattung präsentiert wurde. Als grundlegende Beispiele hierfür sind etwa die Werke des englischen Gesandtschaftssekretärs Paul Ricaut ${ }^{3}$ und des französischen Reisenden Jean-Baptiste Tavernier ${ }^{4}$ zu nennen.

Den ersten turkophilen Ansätzen in der Reiseliteratur folgten die großen epochalen Werke der Wissenschaft. Die intensive, kritische wissenschaftliche Auseinandersetzungen mit der arabischen Kultur und dem Islam durch westeuropäische Orientalisten - wie Edward Pococke, Simon Ockley ${ }^{5}$, Adriaan Reland ${ }^{6}$, Bartholomé d'Herberlot und Antoine Galland ${ }^{7}$ - spielten eine basal wichtige Rolle für die späteren philanthropischen Diskurse der Aufklärungszeit.

Diese Veränderung ist auch in der Literatur spürbar. Die ersten Türkendramen sind zwar bereits im 16. Jahrhundert in Frankreich entstanden, doch das Orientalische diente hier nur als Dekoration, um der Geschichte ein märchenhaftes Ambiente zu verschaffen. Die französische Übersetzung von Tausendundeine Nacht von Antoine Galland ${ }^{8}$ eröffnete schließlich der Literatur diese Welt voll Phantasie, Exotik und Erotik. Die fremde Kultur des Orients wurde auch in den Werken der großen Aufklärer, wie u.a. von Montesquieu oder Voltaire ${ }^{9}$ wachgerufen, um den Zeitgenossen aus der Sicht der Fremden einen kritischen Spiegel vorzuhalten. Der Topos des weisen, toleranten, aufgeklärten Orientalen, wie er in der Figur Sultan Saladin in Lessings Drama Nathan der Weise ${ }^{10}$ oder in Bassa Selim in Mozarts Die Entführung aus der Serail ${ }^{11}$ verkörpert wird, breitete sich aus. ${ }^{12}$ Wie es Susanne Greilich feststellt, tritt auch in den deutsch- oder französischsprachigen volkstümlichen Almanachen im letzten Drittel des 18. Jahrhunderts der Türke als personifiziertes virtutis exemplum auf, dessen Verhalten dem Leser zur Mahnung und Nachahmung dienen sollte. Barmherzigkeit und Mitgefühl, sogar Gottesfürchtigkeit und Besonnenheit in religiösen Dingen machten die Figur des "guten Türken“" zur Musterfigur aufgeklärter Tugenden. ${ }^{13}$

In der ersten Hälfte des 18. Jahrhunderts war also der Wandel im Denken über den Islam, den Orient und die Türken in westlichen Ländern bereits vollzogen, die exotische Welt wurde aber rasch auch in Mittel- und Osteuropa sehr populär. Die positive Türken-Rezeption kam in unterschiedlichen Bereichen der Kultur und des Alltagslebens zum Ausdruck: Literatur, Musik und Architektur, Moden und Kochkunst wurden nachgeahmt, Stoffe, Parfum, Gewürze und Kaffee wurden nach Europa gebracht und gerne verwendet. ${ }^{14}$ 


\section{Türkenmode im westlichen Europa}

Infolge der breiten und vielseitigen Aufnahme und Verarbeitung der osmanischen Kultur wurde diese Teil der politischen, wissenschaftlichen, kulturellen und ästhetischen Diskurse Europas. Dies kann als eine Form der imaginativen Weltaneignung interpretiert werden, die anfangs aber nicht auf einer realen Erfahrung von Fremdheit basierte, sondern eher die Fantasien des Westens über den Luxus und die Exotik des Orients darstellte. ${ }^{15}$

Das Rezeptionsphänomen der sog. Turquerie ist, wie es Maria Elisabeth Pape beschreibt: „eine Domäne derer, die nie den Orient sahen und ihm, der Zeit entsprechend, ein dekoratives, kein ethnographisches Interesse entgegenbrachten. So wurde der türkische Orient auch in den bildenden Künsten zu einer Provinz des Rokoko [...] zum Ausdruck einer gesteigerten gesellschaftlichen Lebensfreude und Repräsentationsentfaltung". ${ }^{16}$

Die barocke Faszination am fernen Osten und die Vorstellungen von Wohlleben, Pracht und freier Liebe wurde in Kreisen der Aristokratie sehr schnell aufgenommen, es entstand die Mode des Exotismus. Die europäischen Orientphantasien haben in den verschiedensten Bereichen des Alltagslebens in Adels- oder Königshöfen Europas Platz gefunden. Türkische Feste wurden organisiert, orientalische Räume eingerichtet, Porträts in türkischem Gewand und in türkischer Szenerie angefertigt.

Bei der Verbreitung der Türkenmode in der höfischen Porträtmalerei oder auf Hoffesten hat die sog. Ferriol-Sammlung, die am öftesten imitierte Bildquelle für die Türken, eine besondere Rolle gespielt. Marquis Charles-Augustin de Ferriol d'Argental, der vom König Ludwig XIV. im Jahr 1699 als französischer Botschafter nach Konstantinopel berufen wurde, beauftragte den damals noch unbekannten jungen Maler des naiven Realismus, Jean-Baptiste Vanmour damit, seine Zeit als Botschafter und vor allem die Welt der Osmanen bildlich zu dokumentieren. Die Kollektion von genau hundert Bildern, die Ferriol anhand der Zeichnungen von Vanmour stechen ließ, wurde sodann mit dem Titel Recueil de Cent Estampes representant differentes Nations du Levant in zwei Bänden, in den Jahren 1714 und 1715, in Paris herausgegeben. Später wurde diese mehrmals neu aufgelegt und in zahlreiche Sprachen übersetzt. Die Bilder von Vanmour avancierten bald zur primären Bezugsquelle der Ikonographie über die Türken, sie waren das wichtigste visuelle Wissen der Zeit über den Orient im 18. Jahrhundert. ${ }^{17}$

Die Stiche zeigen aber nicht nur die höchsten Würdenträger des türkischen Hofstaates - wie den Sultan, den Großwesir oder den Janitscharenführer, sondern auch das einfache Volk des großen Reiches. Unter den einhundert Porträts sind 62 türkische, sowie 10 griechische, 6 afrikanische, 4 armenische, 3 jüdische und je 2 tatarische, persische, albanische, indische, französische und ungarische Porträts 
zu finden. Besonders interessant sind die Bilder, die die Damen des Hofes bei alltäglichen Beschäftigungen wie beim Spielen oder Tanzen, bei der Handarbeit oder einfach lässig am Diwan liegend, mit Pfeife oder mit einem Instrument in der Hand, darstellen. Die großen Fenster zum üppigen Garten, die bunten Vorhänge und Teppiche, die niedrigen Liegesofas mit großen Kissen sind typische Elemente der orientalischen Interieurs. ${ }^{18}$

Vanmours Figuren wurden sehr oft nachgeahmt. Kostümbücher, Porzellane oder Malereien (wie von François Boucher, Francesco und Giovanni Antonio Guardi oder William Hogarth), aber auch die Ikonographie der Odaliske, der exotischen Frau des Harems, und die orientalisierende Aktmalerei des 19. Jahrhundert (unter anderem die Bilder von Jean-Auguste- Dominique Ingres) werden auf die Ferriol-Sammlung zurückgeführt. In ihrer Rezeption verdeutlichen sich beispielhaft die thematische Geschlossenheit und die darstellerische Vielfalt der Turquerie. ${ }^{19}$

Der Recueil Ferriol fand bald direkte Nachahmer, die Türkisierung europäischer Themen etablierte sich auch in der Porträtmalerei. Die ersten Hofporträts im türkischen Kleid sind aus den 1730er-Jahren bekannt. Diese Art der Repräsentation wurde nach der zweiten türkischen Gesandtschaft von 1742 in Versailles zu einer Modewelle und ließ sich bis in die 1780er-Jahre verfolgen. Die exotischen Porträts à la Turque waren modisch-modern und zeitlos zugleich, und gaben den Auftraggebern, ähnlich wie auch durch andere traditionelle Verkleidungen - beispielsweise historische oder mythologische Kostüme - eine Distanz zu den Zeitgenossen und gleichzeitig eine gute Gelegenheit zur Selbstrepräsentation. ${ }^{20}$

Der Anlass zur Verbreitung der orientalischen Herrscherporträts war also der türkische Gesandte Said Pascha, der 1742 in Paris von einem der führenden Gesellschaftsmaler, Jacques-André-Joseph-Camelot Aved, porträtiert wurde. Das Gemälde wurde vor allem in den adeligen Kreisen begeistert aufgenommen, Aved avancierte zum begehrten Porträtisten von Diplomaten, Reisenden, somit hochgestellten Persönlichkeiten, die eine engere Beziehung zum Orient hatten.

Die orientalischen Hofporträts wurden in den 1750er-Jahren durch die Bildnisse der Marquise de Pompadour von Charles André van Loo (1705-1765, genannt Carle van Loo oder auch Carle Vanloo) europaweit bekannt. Vanloo stellte die Mätresse des französischen Königs Ludwig XV. in seinen Bildern Une sultane prenant du café, Deux sultanes travaillent à la tapisserie und Une sultane jouant un instrument à cordes als Sultanin in unterschiedlichen Interieurszenen dar. Die Bilder zeigen die Marquise in europäischen Architekturrahmen mit türkischen Dekorationselementen, wie sie die Zeit mit Plaudern, Musizieren und Kaffee trinkend verbringt. Die Hofdame wird auf den Bildern im türkischen Kostüm des Recueil Ferriol in märchenhafter orientalischer Pracht präsentiert. $^{21}$ 


\section{Turkophilie in Wien}

Mit zwanzig bis dreißig Jahren Verspätung etablieren sich die oben beschriebenen aufgeklärten positiven Vorstellungen vom ,Fremden“ auch in der Adels- und hohen Bürgerkultur der Habsburgermonarchie. Zunächst vor allem im Theater, später auch in der bildenden Kunst, dies allerdings in Menge und Aufwand nicht mit Frankreich vergleichbar. ${ }^{22}$

Der Kaiserin war vermutlich die westeuropäische Mode des Exotismus bewusst, als sie sich mehrmals vor orientalischen Kulissen porträtieren ließ. Ihre Bildnisse sind frühe Ausnahmen der Turquerie im östlichen Teil Europas. ${ }^{23}$ Das erste orientalische Porträt der damals erst 26-jährigen Maria Theresia wurde von Martin van Meytens im Jahr 1743 angefertigt und zeigt sie im Kostüm einer türkischen Haremsdame. Ein Jahr später entstand ein zweites Bildnis alla turca: der berühmte reisende Maler, Jean-Étienne Liotard porträtierte die spätere Monarchin zusammen mit ihrer ältesten Tochter, Maria Anna, in türkischem Gewand.

Liotard hatte zwischen 1737 und 1742 fünf Jahre in der Türkei verbracht, wobei er vom orientalischen Leben so stark beeindruckt war, dass er sich durch einen Bart und türkische Kleidung ein authentisch orientalisches Aussehen verschaffte. Bei seiner Rückkehr nach Europa erregte er mit seiner Erscheinung so großes Ansehen, dass er bald nur noch als ,le peintre turc“ bezeichnet wurde. Der Schweizer Pastell- und Miniaturmaler, der später als reisender Maler durch ganz Europa wanderte und mit unzähligen höfischen Aufträgen reüssierte, hatte stark dazu beigetragen, dass das Porträt im türkischen Kostüm zu einer europaweiten Modeerscheinung wurde. ${ }^{24}$

Sein legendärer Ruhm begann 1743 tatsächlich in Wien, wo er der Lieblingsmaler Maria Theresias wurde. Sein Erfolg am Wiener Hof war so groß, dass er wegen der zahlreichen Porträtbestellungen von Persönlichkeiten des Hofes und Hochadels bald eine eigene Werkstatt einrichten musste. Liotard schuf während seiner Wien-Aufenthalte zahlreiche Porträts der Kaiserin. ${ }^{25}$

Das Bildnis mit dem Titel Maria Theresia und ihre Tochter Maria Anna in türkischen Gewändern ist 1744 entstanden, für seine Anfertigung könnte laut Maria Elisabeth Pape vielleicht ein Karneval den Anlass gegeben haben. ${ }^{26}$ Insgesamt wurden davon zwölf Radierungen im Stil der Ferriol Sammlung fertiggestellt, die verschiedenen Repliken wurden an die weite Habsburger Verwandtschaft verschenkt. Die Beliebtheit dieser Darstellung wird auch dadurch unterstrichen, dass Maria Theresia von Liotard noch eine weitere Miniatur bestellte, die sie ebenfalls in türkischen Kleidern zeigt. ${ }^{27}$

Wenn wir die Herrscherporträts alla turca (unter anderen auch jene von Maria Theresia) betrachten, merken wir einerseits den starken Einfluss der Ferriol Sammlung, andererseits aber auch eine Mischung aus europäischen und türkischen bzw. orientalischen Kompositionselementen: wie auf Liotards Gemälde 
der sehr dominante türkische Teppich oder die für die Damen gewählten Accessoires, die Liotard aus Konstantinopel mitgebracht haben $\operatorname{mag}^{28}$ - etwa die Feder im Haar und der Kaftan, die sich zum Teil zwischen die Kleidungsstücke der klassischen Hoftracht mischen. Dies ermöglicht dem Betrachter, zwischen vertrauter Situation und orientalischer Verfremdung zu vermitteln und das ganze als dekoratives Spiel zu genießen. ${ }^{29}$

\section{Höfische Türkenfeste}

Ein weiteres wichtiges Element der höfischen Repräsentation waren Festlichkeiten, die auf orientalische Art zelebriert wurden. „Feste spielten im Identitätskonstrukt der Eliten der Frühen Neuzeit eine zentrale Rolle. [...] Der Einsatz der verschiedenen Künste in dem Gesamtkunstwerk Fest diente dem Ausdruck der Macht und der Bestärkung und täglichen Bestätigung der hierarchischen Machtverhältnisse der Höfe. Darüber hinaus kam dem Fest sowohl im internationalen Rahmen als auch auf lokaler Ebene eine Funktion in der Konkurrenz der Staaten bzw. der Adeligen des Hofes zu.“30

Über die orientalischen Musik-, Balett- und Theatervorstellungen hinaus wurden regelmäßig türkische Umzüge, Mummereien, Caroussel-Spiele organisiert. In Frankreich war die Kostüm-Turquerie vor allem im Rahmen von Balletten, Turnieren und zu Karneval schon seit Ende des 16. Jahrhunderts verbreitet, aber im 18. Jahrhundert war das türkische Kostüm auch aus den Festlichkeiten des Habsburger Hofes nicht mehr wegzudenken.

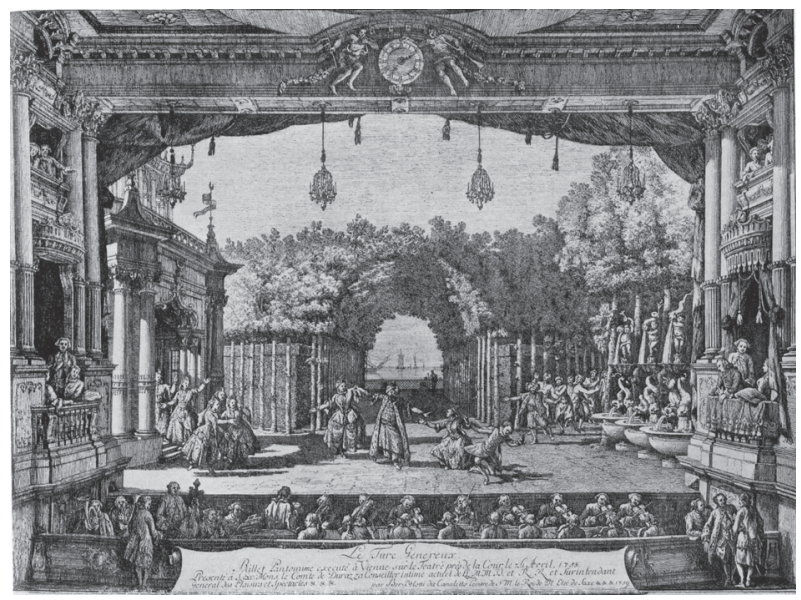

Abbildung 1:

Bernardo Bellotto, genannt Canaletto, Szenenbild aus ,Les Turcs généreux” im Wiener Burgtheater, Wien 1758. 
Die schwärmerische Faszination für den exotischen Orient im höfischen Bereich fand ihren Höhepunkt bereits 1719 in den Hochzeitsfeierlichkeiten der Vermählung von Erzherzogin Maria Josepha von Österreich, der ältesten Tochter des verstorbenen Kaisers Joseph I., mit dem sächsischen Kurprinzen Friedrich August II. (später als August III. König von Polen und Großherzog von Litauen). Die umfangreichen Hochzeitsfeierlichkeiten galten als Glanzstück der Türkenmode im Kreis des Habsburger Hofes.

Das über einen ganzen Monat währende Fest, das vom Vater des Bräutigams, von August dem Starken (auch Hercules Saxonicus genannt), organisiert wurde, sollte einerseits die neue, enge familiäre Verbindung zum Kaiserhaus und andererseits seinen Anspruch auf eine bedeutende Stellung im Reich verdeutlichen. Die persönliche Zuneigung August des Starken zu den zeitgenössischen exotischen Modewellen zeigte sich deutlich: Sein erstes „Türkisches Fest" hatte er bereits 1715 in Warschau gefeiert. Orientalische Feste sowie türkische Maskeraden und Aufzüge gehörten also mittlerweile schon zur Tradition am sächsischen Hof. Das prächtige, orientalische Hochzeitfest kann man aber nicht nur mit dem persönlichen Geschmack des Königs erklären, es hatte auch eine politische Relevanz: Man kann darin auch den Versuch des Wettiners sehen, seine Verbundenheit mit dem Kaiser zu zeigen, der Europa an vorderster Front gegen die Osmanen verteidigte. Wie Elisabeth Mikosch betont, ließ sich August der Starke gern als Türkenbezwinger zeigen, auch wenn seine eigene Teilnahme an den Kämpfen gegen die Türken nur wenig ruhmreich war. ${ }^{31}$

Das große Hochzeitsbankett fand im August 1719 in Wien statt. Zu den geladenen Gästen zählte auch Ibrahim Pascha, der türkische Großbotschafter mit seiner Begleitung, der sich gerade wegen des Friedensschlusses von Passarowitz (1718) in Wien aufhielt. ${ }^{32}$ In Johann Christian Lünigs Theatrum Ceremoniale wird über den Empfang des Botschafters so berichtet:

„[...] der Ambassadeur [wird] von dem Schencken und Hof-Junckern empfangen, und die große Treppe, welche von beiden Seiten mit 100 Schweizern in ihren Parade-Kleidern besetzet ist, hinauf geführet. Oben an der Treppe erwartet ihn der Königl. Schloß-Hauptmann, und in dem erstem Vorgemach der Ober-Marschall mit den Cammer-Junckern, in dem Gemach vor dem Audienz-Saal aber der Ober-Cämmerer, welcher von denen gesammten würdlichen Cammer-Herren umgeben ist, und ihn in der Mitte solches Zimmers empfängt und complimentiret, sodann aber den Audienz-Saal geführet wird. Die 3. Reverenzien des Ambassadeurs und die dreifache Danksagungs-Grüße von Seiten Ihrer Königl. Maj. Geschehen [...]. Wenn nun beiderseits Anrede und Antwort gegen einander geschehen, [...] der Ambassadeur so dann nochmals tractiret und endlich in sein Quartier gebracht." ${ }^{\text {"33 }}$ 
Die Hochzeitsfeierlichkeiten wurden einen Monat später in Dresden, im ehemaligen Italienischen Garten gefeiert, den August der Starke zum „türkischen Garten“ und „türkischen Palast“ umwandeln ließ: Türkisches Kunsthandwerk, wie Teppiche und Goldschmiedearbeiten, wurden zur Ausschmückung herbeigeschafft. Palais und Garten, die später bei dem preußischen Bombardement von Dresden schwer beschädigt und nicht wieder hergerichtet wurden, hat August dem Chronisten Iccander (Johann Christian Crell) zufolge am Tag der „Fête des Turques" der Braut geschenkt. ${ }^{34}$

Die Erzherzogin reiste mit dem Schiff an und wurde mit türkischer Pracht empfangen: Es wurden neunzehn türkische Zelte am Ufer aufgestellt, die abendliche Tafel wurde in Form eines Halbmonds aufgestellt. In Anlehnung an die osmanischen Elitetruppen wurden Janitscharen als Festgarde berufen. Für die Feierlichkeiten wurden 315 Infanteristen ausgesucht, die jung, ansehnlich, von gleicher Größe sein sollten und sich einen „türkischen Schnurrbart“ wachsen lassen mussten. ${ }^{35}$

Die größte Attraktion des Festes war ein in der Grotte im Zwinger aufgebautes Wachsfiguren-Kabinett, die „Circle Ottoman“, das das Serail, den Sultan in seinem Harem, darstellen sollte. Die 16 lebensgroßen Figuren im Stil der Ferriol-Sammlung wurden vom Bildhauer Jean Joseph Vinache fertiggestellt. Das Kabinett zeigte eine stereotypenhafte Situation, in der der Sultan in der Mitte des Raumes auf dem mit kostbaren türkischen Teppichen ausgelegten Boden sitzt und von Sklavinnen und Sklaven mit Musik und Tanz unterhalten wird. Die Szenerie wurde den interessierten Gästen von zwei als Armenier verkleideten französischen Komödianten mit Zeigestöcken erklärt. ${ }^{36}$ Diese Serail-Komposition wurde nach dem Hochzeitsfest in die Rüstkammer im Zwinger gebracht, wo sie noch bis 1800 besichtigt werden konnte. ${ }^{37}$

Auch zu Zeiten Maria Theresias wurden einige Feste mit Türkenthematik durchgeführt. Die wichtigste Quelle über die weltlichen Feste des Habsburger Hofes sind die Tagebuchaufzeichnungen des Fürsten Johann Joseph Khevenhüller-Metsch, der als Obersthofmeister von Maria Theresia zwischen 1742 und 1776 alle Feste, Theater- und Opernaufführungen detailliert geschildert hat. Seine Aufzeichnungen geben einen Einblick in den Zeitenwandel, von den letzten Ausläufern barocker Lebensformen während der ersten Regierungsjahre Maria Theresias hin zum aufgeklärten Absolutismus Josephs II. ${ }^{38}$

So berichtet Khevenhüller-Metsch über ein türkisches Fest im Jahr 1743, das anlässlich des Namenstages der Kaiserin im Schloss Schönbrunn zelebriert wurde:

„Den 3. [Oktober] kehrte man [...] zurück nach Schönbrunn, allwo [...] nicht allein große Gala angelegt und nebst großen Soupé Bal gehalten, sondern auch das Schloss und der mittere Prospect des Gartens mit vilen 
Lampions illuminiret und von der hiesigen operischen Bande eine Entrée in orientalischer Tracht durch den Garten und hierauf ein Ballet im Saal produciret wurde. ${ }^{\text {(39 }}$

Sowohl die Königin, als auch ihre Schwester Maria Anna und die weiteren Gäste der hohen Aristokratie trugen orientalische Kleidungen. Es wurde eine Festgarde von mehr als hundert ungarischen Infanteristen aufgestellt, die ebenso türkisch gekleidet waren und einen feierlichen Aufmarsch hielten. Das Fest wurde mit einer orientalischen Tanzaufführung eröffnet und von türkischer Musik begleitet. Über dieses Fest wird am 5. Oktober 1743 auch in der Wiener Zeitschrift referiert:

\begin{abstract}
„,...] Ihrer Majestät der Königin [und] Frau Schwester Ertz-Hertzogin Maria Anna [hat sich in Schönbrunn] nebst einer Menge hohen Adels in prächtigen Kleidungen erhoben, und weil das gestrige hohe Namens-Fest des Königl. HM. Ehe-Gemahls Hoheit an einen Freytag einfiele, so wurde vorgestern Schloß Schönbrunn samt dem Lust-Garten mit vielen tausend Lampen, Wax-Kertzen und Wind-Lichtern auf das prächtigste illuminiret, sodann von Opera Täntzern des Königl. privilegirten Theater, in Gefolg von mehr dann hundert Hungarischen Infanteristen in unterschiedlichen Orientalischen Kleidungen, ein curioser Einzug durch den Garten nach dem Saal gehalten, und da von den Opera-Tantzern ein Orientalischer Tantz aufgeführet; wonach sodann später von denen Höchsten Herrschaften der Ball eröffnet wurde. ${ }^{\text {“40 }}$
\end{abstract}

Die politischen und militärischen Erfolge der Habsburger und ihrer internationalen Verbündeten, der neue Status quo auf dem Balkan, brachten also für den deutschsprachigen Teil West-Europas ein neues Lebensgefühl mit sich. Die orientalischen Mummereien, Aufzüge, Bälle, höfischen Feiern und Volksfeste zeigen aber ein sehr ambivalentes Türkenbild, das vor allem der höfischen Repräsentation und der aktuellen politischen Propaganda diente. Die turkophilen Exotismen erfassten in der Habsburgermonarchie nur die soziokulturellen Eliten, die das Land dominierende Volkskultur blieb - wie Grothaus es mehrmals betont - von den mentalen Umwälzungen, die aus Westeuropa übernommen wurden, eigentlich unberührt. ${ }^{41}$

\title{
Volksfeste der triumphalen Allegorie
}

Auf den Festen des Hofes wurden also die Bilder der Turkophobie zum Großteil von der Turkophilie abgelöst, in Kreisen breiterer gesellschaftlicher Schichten waren weiterhin jene Volksfeste in Gebrauch, die neben dem allgemeinen Interesse für die exotische Kultur die jahrhundertealten Motive der triumphalen Allegorie - Besitzergreifung, Unterwerfung, Demystifikation - aufzeigten. 
Das wohl bekannteste dieser Volksfeste ist der Hernalser Eselritt bzw. Türkenritt, der zum Andenken an die ,glückliche Befreiung Wiens“ jährlich am Sonntag nach Bartholomäus aufgeführt wurde. Wann der Spottaufzug zum ersten Mal stattgefunden ist, lässt sich nicht ermitteln, er wurde aber wahrscheinlich gleich nach der türkischen Niederlage an einem Kirchweihtag vorgeführt und blieb dann erhalten. $^{42}$

Caroline Pichler, die das Fest in Hernals als Kind immer miterlebt hat, beschreibt es wie folgt:

\begin{abstract}
„Die Teilnehmer des Zuges, der sich unter militärischer Musik durch das ganze lange Dorf bewegte, waren großenteils ziemlich grotesk maskiert, sie führten einen Esel mit sich, von dem das Fest seinen Namen trug, hinter ihm schritt ein Mensch in gewöhnlicher deutscher Tracht, mit Ketten belastet und von einem Türken geführt, dem auf einer Art Triumphwagen ein sogenanneter Sultan mit einer Sultanin in theatralischem Pomp und hinter ihnen noch viele maskierte Türken folgten" ${ }^{43}$
\end{abstract}

Die Hauptattraktion der Prozession zur Verhöhnung der Osmanen war die Darstellung von Kara Mustafa - als wohlbeleibter Pascha, im schönsten morgenländischen Schmuck -, der verkehrt auf einem Esel ritt und vom Publikum so lange betrunken gemacht wurde, bis er vom Esel fiel. Weiters zogen „Janitscharen“ vorbei, die „Christensklaven“ in Ketten gebunden hatten und junge Mädchen aus dem Publikum herausschnappten, die sich dann freikaufen mussten.

Von Hans Will wird der Festzug detailliert beschrieben:

„Und wenn Tag und Stunde des Festes gekommen waren [...] glühten die Wangen und pochten die Herzen der Kirchtagsgäste in Erwartung. [...] Die Tore wurden geöffnet. Höllischer Lärm, Gelächter und die Klänge der türkischen Musik, der es an Dudelsäcken, Maultrommeln und Geigen nicht fehlte, verkündeten das Herannahen der Spitze des Zuges. An die Musikbande reihte sich gewöhnlich eine Schar jämmerlich gekleideter Christensklaven, die, mit Stricken und Ketten gefesselt, von grimmig aussehenden, bärtigen Janitscharen geführt. [...]

Die wachehaltenden Janitscharen waren die Schrecken der Dorfschönen, denn kam ihnen eine zu nahe, dann wurde sie erbarmungslos in die Reihen der Sklaven gezerrt und erst auf ein Lösegeld in barer Münze oder gar in Küssen wieder freigegeben.

Als nächste Truppe kam eine Schar bewaffneter Türken und ihnen folgte der Hauptspaß des Maskenumzuges: der in der größten Pracht morgenländischen Schmuckes prangende Pascha. Meist selbst von gewaltigem Leibesumfang, saß er auf dem dicksten Esel, den man in der ganzen Umgebung auftreiben konnte, und löste durch sein Aussehen allein schon Lachsalven bei den Zuschauern aus. Und wenn er gar, allen Gesetzen und Regeln des Korans entgegen, dem Weine, der ihm von den einzelnen Weinbauerhäusern herausgereicht wurde, herzhaft zusprach, dann wurden seine Reden und Gebärden immer komischer und er war die Zielscheibe des Spottes und mannigfachster Neckereien. ${ }^{\text {“44 }}$ 
Der Eselritt kann laut Anton Mailly auf eine orientalische Strafform zurückgeführt werden, die vor allem auf Frauen angewendet wurde, die entweder Ehebruch begangen oder ihre Männer geschlagen hatten. Um die „größte Schmach, die eine Frau ihrem Mann antun konnte, zu rächen“, musste die beschuldigte Frau verkehrt auf einen Esel steigen und seinen Schwanz haltend durch den Ort reiten. Erst nach der Entsatzschlacht, die auf Hernalser Gebiet stattgefunden hat, wurde der Umzug auf die Türken angepasst. ${ }^{45}$

Die ersten Quellen, die Kara Mustafa, die Hauptfigur des Festes, auf einem Esel reitend darstellen, stammen aus dem Jahr nach der zweiten Wiener Türkenbelagerung, 1684. Das Flugblatt mit dem Titel Wer suecht, der findt. Deß Türckischen Groß-Vizirs Cara Mustapha Bassa Zuruck-Marsch von Wienn nacher Constantinopel des Wiener Verlegers Leopold Voigt zeigt, wie sich der besiegte Kara Mustapha Pascha auf einem Esel sitzend, weinend, gefolgt von seiner gebrochenen und gedemütigten Armee nach Konstantinopel zurückzieht. ${ }^{46}$

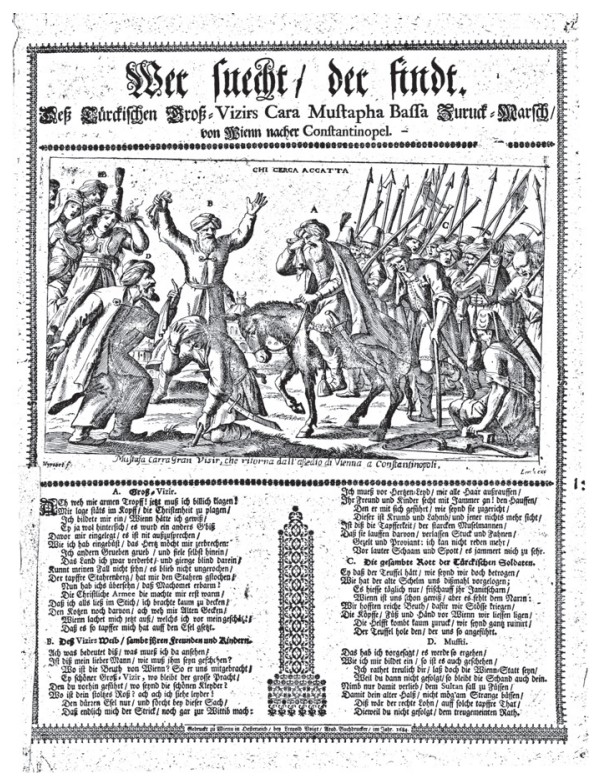

Abbildung 2:

Johann August Corvinus: Die königliche Tafen und Schaubuden beim Merkurfest im Zwinger 1719, nach der Zeichnung von Carl Heinrich Jacob Fehling, nach 1725.

Der Hernalser Eselritt war noch in der Zeit von Maria Theresia gebräuchlich, doch schon Joseph II. zeigte für diese Form repräsentativer Festlichkeiten wenig Verständnis und verbot die Belustigung. Der letzte Aufzug am 25. August im Jahr 1783 wurde jedoch noch unter großem Interesse organisiert - Will, ein Augenzeuge, berichtet darüber wie folgt: 
„Dreyßig Schritte vor dem Schottenthore - innerhalb der Stadt meyne ich - ließ sichs schon gewahrt werden, daß in der Nachbarschaft etwas Festliches vorgehen müsse, denn mein Wagen mußte hinter zehn oder zwölf andern eine hübsche Weile warten, weil die Hereinfuhr einer kaum zählbaren Reihe von Wägen das Thor zu enge machte. [...] Im Vorbeyfahren nun sah ich Bürgersleute, Handwerksburschen, Stubenmädel, gnädige Frauen mit strohenen Sommerhüten [...], Invaliden, Polizeykoporale, alte Weiber, Fleischerknechte mit großen Hunden, Buchdrucker, Artilleristen, Juden, Haiducken, Authoren, Obstweiber, wälsche Würstkrämer, Taglöhner, Philosophen u.s.f., ein jedes auf seine Weise, und mit seiner besondern Phisiognomie von Erwartung und Eifer der Linie zuspazieren. ${ }^{647}$

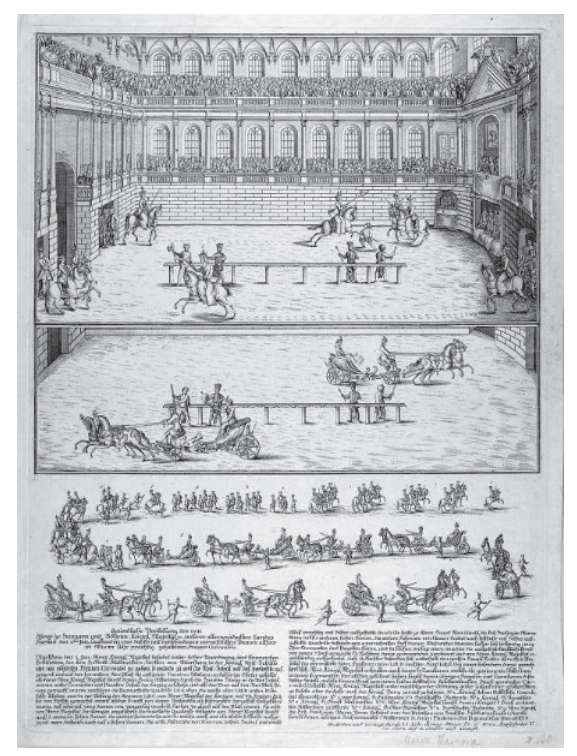

Abbildung 3:

Justus van den Nypoort und Johann Martin Lerch: Wer suecht, der findt. Des

Tuerkischen Groß-Vizirs Cara Mustapha Bassa Zurück-Marsch von Wien nach Constantinopel,

Wien 1684.

Als eine weitere, sowohl im kleinbürgerlichen und bäuerlichen als auch im höfischen Milieu sehr beliebte Gelegenheit, die Osmanen stellvertretend zu besiegen, galt das „,Türkenkopfstechen“, ein Lanzenstechen nach Türkenfiguren und -köpfen aus Holz oder Pappmaché.

Die Geschichte des Türkenkopfstechens geht auf das 16. Jahrhundert zurück, wobei es lange Zeit hindurch die propagandistische Verbreitung des Feindbildes unterstützen sollte. 
Das Theatrum Europaeum beschreibt, wie im Jahr 1661 für einen türkischen Gesandten in Wien im Rahmen eines Karussells anlässlich der Vermählung Leopolds I. mit der Infantin Margarita Theresa der Brauch des Türkenkopfstechens vorgeführt wurde: ${ }^{48}$

\begin{abstract}
„Auf dem Thurnier Platze waren 4 hierzu bereitete Türcken-Köpffe an vier unterschiedlichen Orten aufgestellt, nach welchen die Ritter mit so vielen unterschiedlichen Gewehren rennen mußten, und zwar nach dem ersten mit der Lantze, ihn zu durchspissen. Nach dem andern mit dem Pfeile, ihn zu durchwerffen. Nach dem dritten mit dem Pistol, ihn zu durchwerffen. Nach dem vierdten mit dem Degen / ihn auffzuheben. Die Römische Kayserliche Maj. und beyde HHn. Erzherzogen sahen selbsten mit zu und wol bey 10. oder 12000. andere Menschen.

Man ließ auch den anwesenden Türckischen Gesandten mit seinen Leuten hierzu einladen, damit er daraus abnehmen möchte, was künfftig nechst Göttlicher Hülffe durch dergleichen Ritters-Personen [...] angestellt werden könte. Derselbige sahe auch diesem Spiele mit zu, das aber wolte ihm nicht gefallen, daß man selbiges auf Türcken-Köpffe gerichtet hatte, und solte er gegen einen zum Christenthum bekehrten Türcken mit dem er reden können, ausdrücklich gesagt haben: Sein Großmächtigster Kayser liesse in Siebenbürgen und anderwerts lebendige Christen-Köpffe in genugsamer grosser Anzahl absäbeln, und hier triebe man mit erdichteten Türcken-Köpffen solche unnöthige Kurzweil. War von einem Barbarn sein ganz barbarisches Urtheil.“449
\end{abstract}

Dieses Vergnügen, das vor allem im 17. Jahrhundert beliebt war, wurde sogar von der Kaiserin Maria Theresia selbst ausgeübt. Am 2. Januar 1743, noch vor ihrer Krönung zur Königin von Böhmen ließ Maria Theresia anlässlich des Abzugs der Franzosen und Bayern aus Böhmen ein Fest in der Winterreitschule der Hofburg organisieren.

Während des Festes wurde in der Hofreitschule ein Damenkarussel vorgeführt. Khevenhüller-Metsch zeichnet das Spektakel so auf:

\begin{abstract}
„Den 2. [Januar] ward bei Hoff das [...] Frauen-Carousel sehr prächtig gehalten. [...] Jedermann verwunderte sich, daß alles hierbei so ordentlich und ohne einigen widrigen Zustoß abgeloffen, da doch die Frauen und Hoff-Dames, so zu Pferd und in Birocci mitgerennet, sehr wenig Zeit, sich zu exerciren gehabt, und sonderlich das reutende Frauen-Volck mit schlechten und kaum zur Promenade, geschweige zu dergleichen Exercitien abgerichteten Pferden versehen gewesen. Überhaubt ware das Spectacul seiner Seltsam- und Neuigkeit halber würdig anzusehen; es manglete auch nicht an Magnificence. ${ }^{\text {"50 }}$
\end{abstract}

An dem Damenkarussel übten die Vertreterinnen der Aristokratie, geführt von der Kaiserin, das Türkenkopfstechen aus, und stachen mit Degen auf aufgespieß- 
te, gut ausgearbeitete „Türkenköpfe“. Maria Theresia selbst fuhr mit einigen der teilnehmenden Damen in den Karussell-Wagen durch die Stadt, so dass die Wiener Bevölkerung die Festgesellschaft bestaunen konnte.

Die beschädigten und somit nicht weiter brauchbaren Türkenköpfe wurden später, auf Brunnen oder Häusern befestigt, als Hausschmuck weiterverwendet. Der Türkenkopf als eine Art triumphale Bekrönung über Türbogen oder unter der Dachtraufe entsprach ganz dem barocken Lebensgefühl, theatralische Zeichen der militärischen Siege zu setzen. Obwohl das finstere und abweisende Aussehen der Köpfe auf ein apotropäisches Motiv hindeutet - die Figuren sollten also vor allem Abschreckungs- und Abwehrfunktion haben -, wurden alle Türkenköpfe nach 1683 angebracht, in einer Zeit, in der die Türkengefahr für Wien längst gebannt war. ${ }^{51}$

Das Zedler Universallexikon berichtet bezüglich der Leipziger Messe im Jahr 1684 sogar über den Handel mit gedörrten Türkenköpfen aus der Schlacht um Wien des vorangegangenen Jahres:

\begin{abstract}
„Türcken-Köpfe. (gedörrete) In der Leipziger Neu-Jahres-Messe des 1684sten Jahres nach glücklichem Entsatz der von den Türcken belagerten Stadt Wien kamen einige Kaufleute in Leipzig an, welche mit dergleichen Köpffen damahls handelten, deren sie verschiedene Fässer voll mitbrachten, so theils grausame Gesichter, theils ungewöhnliche Bärte und vielerley Haupt-Haare hatten. Einige waren lang gewachsen; andere hingen kurz abgeschnitten; nachdem nun dieselbige wohl gestaltet oder die Gesichter nicht zersetzt gewesen, wurde das Stück zu 4.6.8. und mehr Reichs-Thalern verkaufft, welche Köpffe hernach zum fortwährenden Andencken des herrlich erfochtenen Sieges in die meisten Königreiche und Lände sind verschicket und auf die vornehmsten Kunst Kammern und Bibliothecken von Europa gekauffet worden. Diese Kaufleute wusten ihre ungemeine Waare mit aufgesteckten Billets zu qualificiren, und den einen Kopf vor dieses, den andern vor jenes Bassa und grossen Türckischen Herren-Kopf auszugeben, da es doch nur etwan eines gemeinen Spahi oder Janitscharens auch etwan gar eines Cameel-Treibers Kopf seyn mochte." ${ }^{.52}$
\end{abstract}

Dieser zeitgenössische Bericht über den Verkauf von Türkenköpfen in Deutschland zeugt von der sich wandelnden und sehr ambivalenten Haltung Europas zu den Türken: ausgehend von einem dominanten Gefühl von Angst und Bedrohung hin zu Spott und Hohn.

Die Kontinuität des Türkenkopfstechens hielt außerordentlich lange an: Noch bis in die erste Hälfte des 19. Jahrhunderts wurden auf Turnieren und Karussellen, bei Reit- und Fechtübungen Zielfiguren türkisch dargestellt. Noch während des Wiener Kongresses im Jahr 1814 wurde ein zeremonielles Türkenstechen organisiert, bei dem reitende Aristokraten auf Türkenköpfe und Orientalenbüsten einstachen. An einem Galafest nahm August Graf de la Garde-Chambonas teil und merkte an: „Ohne Zweifel erhielt man auf diese Weise ehemals den Haß 
der deutschen Krieger gegen ihre räuberischen, unversöhnlichen Feinde, die Türken. ${ }^{\text {" }} 33$ Es war dies eine Form der Belustigung, die - wie Karl Vocelka betont - in Westeuropa zu dieser Zeit schon längst nicht mehr denkbar war. ${ }^{54}$

\section{Fazit}

Die Schaugefechte und triumphalen Turnieraufzüge gaben sowohl der Aristokratie als auch den niedrigeren gesellschaftlichen Schichten Gelegenheit, eine metaphorische Aufarbeitung der Türkenkriege durchzuführen und dabei auch das Identitätsbild des heldenhaften Volkes im Sinne von nation building zu stärken. Dass diese Aktivitäten durch Friedenszeiten hindurch und noch bis zum 19. Jahrhundert ausgeübt wurden, zeugt von einer sich stark wandelnden Interpretation osmanischer Kultur, geprägt von einer ambivalenten Haltung aus Furcht und Bewunderung, Mythisierung und Verniedlichung.

Das osmanische Feindbild des Mittelalters und der Frühen Neuzeit mit seinen zahlreichen, in vielen europäischen Literaturen und Kulturen weit verbreiteten Topoi der Grausamkeit, Heimtücke, Blutrünstigkeit oder Gottlosigkeit hatte eine tiefgründige eschatologische Bedeutung: Ausgehend von der Auffassung Luthers wurden die Türken nicht nur als eine weltliche Macht, sondern als eine göttliche Strafe, eine „Geissel oder Zornesrute Gottes“ (flagellum et virga Dei) für die Sünden der Christenheit interpretiert. ${ }^{55}$ Diese heilsgeschichtliche Auslegung der Osmanen hat in mitteleuropäischen Ländern für eine starke archetypische Fixierung und Prägung der Feindvorstellungen gesorgt. ${ }^{56}$

Wie Grothaus es betont, „mündeten sowohl die profane als auch die heilsgeschichtliche Dimension des Feindbilds im mentalitätsgeschichtlichen Grundkonzept konsequent im Sieg über den Feind. Der Türkentriumph war zweifellos eines der wichtigsten propagandistischen Themata der kaiserlichen Propaganda zwischen dem 16. und 18. Jahrhundert. Die Stilmittel reichten dabei von simplen Waffentropaia bis hin zu großzügigen, theatralischen Inszenierungen, in denen entweder Schlachten, wie der Entsatz von Wien 1683, oder die Habsburger allgemein als Herculier gefeiert wurden." ${ }^{\text {(57 }}$

Überblickt man die Literatur, Kultur und Kunst im späten 17. und frühen 18. Jahrhundert, wird es deutlich, dass das kulturelle Interesse für den Orient, die Anspielungen und Nachahmungen des exotischen Feindes, also die Türkenmode, in den östlichen Ländern Europas, somit zum Teil in der österreichischen Habsburgermonarchie, nur ein Teilaspekt des Exotismus wurde, der vor allem der höfischen bzw. adeligen Repräsentation diente. Die langen Türkenkriege haben in diesen Ländern zur Ausbildung und Verstärkung eines expansiven Sendungsbewusstseins und zu einer starken Tendenz der Verharmlosung und Demythisierung des ehemaligen Feindes geführt. 


\section{Anmerkungen}

1 Vgl. u.a. András Forgó, Überlegungen zum Wandel des Osmanenbildes im Königreich Ungarn der Frühen Neuzeit - In: Gabriele Haug-Moritz, Ludolf Pelizaeus (Hrsg.): Repräsentationen der islamischen Welt im Europa der Frühen Neuzeit, Münster 2010, 75-94, hier 87-88. / Cornelia Kleinlogl, Exotik-Erotik. Zur Geschichte des Türkenbildes in der deutschen Literatur der frühen Neuzeit (1453-1800), Frankfurt am Main 1989, 301-415. / Marc-Oliver Rehrmann, Ehrenthron oder Teufelsbrut: Das Bild des Islams in der deutschen Aufklärung, Zürich 2001. I Asli Cirakman, From the „, Terror of the world" to the „Sick Man of Europe“. European Images of Ottoman Empire and Society from the Sixteenth Century to the Nineteenth, New York 2005, 105-183. / Barbara Schmidt-Haberkamp, Grenzüberschreitungen in kosmopolitischer Zeit - In: Europa und die Türkei im 18. Jahrhundert, Bonn 2011, S. 9-27. / Volker C. Dörr, Wollust und Macht im Harem: Das Bild des Türken in Trivialdramen Ifflands und Kotzebues, 63-81. / Dirk F. Passmann: Mahometthe Great and Jonathan Swift: The Story of Irene and the Image of the Turk in Early Eighteenth-Century English Literature, 95-109. / Davor Dukic, Das Türkenbild in der kroatischen literarischen Kultur des 18. Jahrhunderts, 109-121. / Susanne Greilich, „Alles, was sich bei den Türken ereignet, ist bedeutend": Turkophilie und Turkophobie in der populären Presse, 177-191.

2 Maximilian Grothaus, Zum Türkenbild in der Kultur der Habsburgermonarchie im Ostalpenraum zwischen dem 16. und 18. Jahrhundert - In: Karl Vocelka (Hrsg.): Mitteleuropa und die Türken: Politische und kulturelle Beziehungen zwischen zwei Kulturkreisen, Wien 1983, 57-67, hier 62.

3 The history of the present state of the Ottoman Empire, London 1682.

4 Les six voyages de Jean Baptiste Tavernier, écuyer baron d'Aubonne, qu'il a fait en Turquie, en Perse, et aux Indes, pendant l'espace de quarante ans, \& par toutes les routes que l'on peut tenir: accompagnez d'observations particulieres sur la qualité, la religion, le gouvernement, les coutumes \& le commerce de chaque païs; avec les figures, le poids, \& la valeur de monnoyes qui y ont court, Paris 1676.

5 The History of the Saracens, London 1708-1718.

6 u.a.: De religione Mohammedica libri duo, Utrecht 1705, 1717.

7 Bibliothèque orientale, ou dictionnaire universel contenant tout ce qui regarde la connoissance des peuples de l'Orient [zus. mit Antoine Galland], Paris 1697.

8 Les mille et une nuits, contes arabes traduits en français par Galland, Paris 1704-1705.

9 u.a. Montesquieu, De L'esprit des Loix, Genf 1748 ; Voltaire, Zaïre, Uraufführung am 23. August1732, Paris.

10 Gotthold Ephraim Lessing, Nathan der Weise, [Berlin] 1779.

11 Wolfgang Amadeus Mozart, Die Entführung aus dem Serail, Uraufführung am 16. Juli 1782, Wien.

12 Vgl. Karl-Ulrich Syndram, Der erfundene Orient in der europäischen Literatur vom 18. bis zum 20. Jahrhundert - In: Gereon Sievernich, Hendrich Budde (Hrsg.): Europa und der Orient 800-1900, Berlin 1989, 324-341.

13 Susanne Greilich: „Alles, was sich bei den Türken ereignet, ist bedeutend“: Turkophilie und Turkophobie in der populären Presse - In: Barbara Schmidt-Haberkamp (Hrsg.): Europa und die Türkei im 18. Jahrhundert, Bonn 2011, 184-186. Siehe auch: Susanne Greilich, Orientbilder in französisch- und deutschsprachigen Volksalmanachen des achtzehnten und neunzehnten Jahrhunderts. In: Rückert-Studien. Jahrbuch der Rückert-Gesellschaft, XV (2003), 59-78. I Susanne Greilich, Les figures de l'exotisme dans les almanachs populaires européens aux XVIIIe-XIXe siècles - In: Hans-Jürgen Lüsebrink, York-Gothart Mix, Jean-Yves Mollier et Patricia Sorel (Hrsg.): Les Lectures du peuple en Europe et dans les Amériques, Histoire Culturelle, Bd. 4, Bruxelles 2003, 271-280. / Susanne Greilich, La Représentation des peuples et des cultures 
orientaux dans l'almanach du Messager boiteux au siècle des Lumières. In: Françoise Lotterie, Darrin M. McMahon (Hrsg.): Les Lumières européennes dans leurs relations avec les autres grandes cultures et religions, Etudes internationales sur le dix-huitième siècle, Bd. 5, Paris 2002, 191-211.

14 Barbara Schmidt-Haberkamp: Europa und die Türkei im 18. Jahrhundert: Grenzüberschreitungen in kosmopolitischer Zeit. In: Barbara Schmidt-Haberkamp (Hrsg.): Europa und die Türkei im 18. Jahrhundert, Bonn 2011, S. 9-27, hier 10. / Andrea Seidler: Wie aus Angst Interesse wird: das volatile Bild der Osmanen in der zentraleuropäischen Literatur des 17. und 18. Jahrhunderts. In: Matthias Schmidt, Daniela Finzi (Hrsg.): Narrative im (post)imperialen Kontext. Literarische Identitätsbildung als Potential im regionalen Spannungsfeld zwischen Habsburg und Hoher Pforte in Zentral- und Südosteuropa, Tübingen 2015, S. 13-24, hier S. 18-19.

15 Vgl. Barbara Schmidt-Haberkamp, Grenzüberschreitungen in kosmopolitischer Zeit. - In: Barbara Schmidt-Haberkamp (Hrsg.), Europa und die Türkei im 18. Jahrhundert, Bonn 2011, S. 9-27, hier 12.

16 Maria Elisabeth Pape, Turquerie im 18. Jahrhundert und der „Recueil Ferriol “ - In: Gereon Sievernich, Hendrich Budde (Hrsg.): Europa und der Orient 800-1900, Berlin 1989, 305-319, hier 305. Vgl.: Maria Elisabeth Pape, Die Turquerie in der bildenden Kunst des 18. Jahrhunderts, Universität zu Köln 1987.

17 Ebd.

18 Pape, 1989, 307-308.

19 Dieter Marcos, Jean-Babtiste Vanmour: Maler zwischen Orient und Okzident - In: Beate Dorfey und Mario Kamp (Hrsg.), ,Die Türken kommen”, Exotik und Erotik: Mozart in Koblenz und die Orient-Sehnsucht in der Kunst, Katalog zur Ausstellung im Mittelrhein-Museum Koblenz, Koblenz 2006, 57-65.

20 Pape, 1989, 312-313.

21 Pape, 1989, 314.

22 Maximilian Grothaus, Zum Türkenbild in der Adels- und Volkskultur der Habsburgermonarchie von 1650 bis 1800 - In: Das Osmanische Reich und Europa 1683 bis 1789: Konflikt, Entspannung und Austausch (=Wiener Beiträge zur Geschichte der Neuzeit, 10/1983), Wien, 1983, 63-89, hier 86-87.

23 Ebd.

24 Walter Koschatzky, Jean-Etienne Liotard in Wien - In: Walter Koschatzky (Hrsg.), Maria Theresia und ihre Zeit: Zur 200. Wiederkehr des Todestages. Ausstellung, 13. Mai bis 26. Oktober 1980, Wien, Schloss Schönbrunn. Salzburg, Wien 1980, 310.

25 Ebd.

26 Pape, 1987, 105.

27 Kerstin Tomenendal, Das türkische Gesicht Wiens: Auf den Spuren der Türken in Wien, Wien, Köln, Weimar, 2000, 48.

28 Koschatzky, 1980, 110.

29 Pape, 1989, 311.

30 Karl Vocelka, Glanz und Untergang der höfischen Welt: Repräsentation, Reform und Reaktion im Habsburgischen Vielvölkerstaat - In: Herwig Wolfram (Hrsg.): Österreichische Geschichte 1699-1815, Wien 2001, 185-186.

31 Elisabeth Mikosch, Ein Serail für die Hochzeit des Prinzen: Turquerien bei den Hochzeitsfeierlichkeiten in Dresden im Jahre 1719 - In: Claudia Schnitzer, Im Lichte des Halbmonds. Das Abendland und der türkische Orient, Leipzig 1995, 235-243, hier 235.

32 Mikosch, 1995, 236.

33 Johann Christian Lünig, Theatrum Ceremoniale, 2 Bde., Leipzig 1719-1720, Bd. 2, 491.

34 Mikosch, 1995, 238.

35 Mikosch, 1995, 237-238. 
36 Mikosch, 1995, 242.

37 Ebd, Kat.-Nr. 324, S. 260.

38 Elisabeth Großegger, Theater, Feste und Feiern zur Zeit Maria Theresias 1742-1776: Nach den Tagebucheintragungen des Fürsten Johann Joseph Khevenhüller-Metsch, Obersthofmeister der Kaiserin, Wien 1987. Vgl. Vocelka 2001, 187-188.

39 Großegger, 1987, 17.

40 Wiener Zeitschrift, Sa. 5. Oktober 1743, 7.

41 Grothaus, 2002, 112.

42 Anton Mailly, Der Hernalser Eselritt - In: Wiener Zeitschrift für Völkerkunde, XXXII (1927), 1-6. Vgl. Tomenendal, 2000, 28.

43 Zitiert nach: Der Eselritt in Hernals, Ein Alt-Wiener Türkenfest - In: Kleine Volkszeitung, Mittwoch 30. August 1933, 6-7.

44 Hans Will, Der Eselritt in Hernals - In: Hernals. Ein Heimatbuch für den 17. Wiener Gemeindebezirk, hrsg. von Hernalser Lehrern, Wien, 1924, 159-161, hier 160.

45 Anton Mailly, Der Hernalser Eselritt - In: Wiener Zeitschrift für Völkerkunde, XXXII (1927), $1-6$, hier 5 .

46 Mailly, 1927, 5.

47 Will, Der Eselritt zu Hernals vom Jahre 1783 den 25. August, Ein Pendant zu Herrn Nikolais Reisebeschreibung, Wien 1783, 6 und 8-9.

48 Vgl. Karl Teply, Türkische Gesandtschaften nach Wien 1488-1792 - In: Österreich in Geschichte und Literatur, Bd. 20 (1976), 14-33.

49 Theatrum Europaeum, Bd. 9, 1672, 548.

50 Großegger, 1987, 3.

51 Reingard Witzmann, Türkenkopf und Türkenkugel - In: Robert Weissenberger (Hrsg.), Die Türken vor Wien. Europa und die Entscheidung an der Donau 1683, Wien 1983, 282.

52 Johann Heinrich Zedler (Hrsg.), Grosses vollständiges Universal-Lexicon [...], Bd. 45, Leipzig/Halle 1745, 1701.

53 Maximilian Grothaus, Der „Erbfeind christlichen Namens”, Studien zum Türken-Feindbild in der Kultur der Habsburgermonarchie zwischen 16. und 18. Jahrhundert, (Dissertation) Graz 1986, 163.

54 Vocelka, Grothaus, 1983. Vgl. Vocelka, 2001, 193.

55 Eine wichtige Quelle für diese Vorstellung ist die vor dem Hintergrund der arabisch-islamische Expansion im letzten Jahrzehnt des 7. Jahrhunderts entstandene Revelationes des syrischen Autors Pseudo-Methodius. Nachdem die eschatologische Schrift im 8. Jahrhundert ins Lateinische übersetzt worden waren, verbreitete sich in der europäischen Literatur der Topos der Muslime als apokalyptisches Volk, das von Gott wegen der Sünden der Menschheit geschickt wurde. Vgl. Fodor Pál, The View of the Turk in Hungary: the Apocalyptic Tradition and the Red Apple in Ottoman-Hungarian Context. - In: Benjamin Lellouch und Stéphane Yerasimos (Hrsg.), Les traditions apocalyptiques au tournant de la chute de Constantinople, Paris 2000 (Varia Turcica 33), 99-131, hier v.a. 104. / Hans Ulrich Hofmann, Luther und die Johannes-Apokalypse. Dargestellt im Rahmen der Auslegungsgeschichte des letzten Buches der Bibel und im Zusammenhang der theologischen Entwicklung des Reformators, (Phil. Diss.), Tübingen 1982 (= Beiträge zur Geschichte der biblischen Exegese 24), 496. / Carl Göllner, Die Türkenfrage in der öffentlichen Meinung Europas im 16. Jahrhundert (= Turcica. Bd. 3), Bucureşti -BadenBaden 1978 (= Bibliotheca bibliographica Aureliana 70), 176.

56 Maximilian Grothaus, Vom Erbfeind zum Exoten: Kollektive Mentalitäten über die Türken in der Habsburger Monarchie der frühen Neuzeit. - In: Inanc Feigl et. al. (Hrsg.), Auf den Spuren der Osmanen in der österreichischen Geschichte, (= Wiener Osteuropa Studien, Band 14) Frankfurt am Main, Wien, 2002, 99-115, hier 103.

57 Ebd. 